\title{
Associação do Anticorpo Anticitrulina e Gravidade da Artrite Reumatóide
}

\section{Association of Anti-Cyclic Citrullinated Peptide Antibody and Severe Rheumatoid Arthritis}

\author{
Aldifran Ferreira da Silva ${ }^{(1)}$, Afonso Napoleão $\operatorname{Matos}^{(2)}$, Áurea Maria Santana Lima ${ }^{(3)}$, Elízia Fernandes \\ Lima $^{(4)}$, Maria Isabel Campos de Couto Correa ${ }^{(5)}$, Edgar M. Carvalho ${ }^{(6)}$
}

\section{RESUMO}

Objetivos: Avaliar a associação do anticorpo antipeptídeo citrulinado cíclico (anti-CCP) com distintos parâmetros clínicos, sorológicos e radiológicos. Métodos: Anti-CCP e fator reumatóide (FR) foram pesquisados no soro de 100 pacientes com artrite reumatóide (AR). A atividade da doença foi definida por meio de um índice combinado compreendendo cinco parâmetros: número de juntas inflamadas, número de juntas doloridas, rigidez matinal, escala visual analógica (EVA) de dor e velocidade de hemossedimentação (VHS). A capacidade funcional foi medida pelo índice HAQ (Health Assessment Questionnaire) e a classe funcional foi determinada mediante aplicação dos critérios revisados do American College of Rheumatology (ACR), de 1991. Erosão e pinçamento articular foram graduados pelo índice de Sharp modificado. A análise estatística empregou os testes do Qui-quadrado, Mann Whitney e Kruskal-Wallis. Resultados: Nenhum dos dois anticorpos demonstrou associação significativa com atividade da doença, sexo, idade de início da doença, presença de nódulos subcutâneos e síndrome de Sjögren. A média de idade foi significativamente menor nos pacientes com AR anti-CCP positivos. A positividade para o FR e anti-CCP foi maior nos pacientes com AR com menos de 50 anos em comparação com os pacientes com mais de 50 anos. A AR de início recente ( $<2$ anos) não se associou a uma maior prevalência de soropositividade para o anti-CCP e FR. O anti-CCP apresentou uma correlação positiva moderada com o FR. Houve uma correlação direta entre o anti-CCP e a VHS e a proteína C reativa (PCR). Houve também uma correlação direta entre o FR, a VHS e a PCR. A classificação funcional dos critérios revisados do ACR de 1991 não se associou a qualquer dos dois auto-anticorpos. $\mathrm{O}$ índice de HAQ não se correlacionou com a atividade da doença, duração da doença, positividade do FR e atividade medida pela PCR, mas associou-se nitidamente à positividade do anti-CCP e à atividade medida pelo

\section{ABSTRACT}

Objective: Evaluate the association of Anti-Cyclic Citrullinated Peptide Antibody (anti-CCP) with distinct clinic, serological and radiological parameters. Methods: anti-CCP and rheumatoid factor $(R F)$ were determined in the serum of 100 patients with rheumatoid arthritis $(R A)$. Disease activity was defined by means of a combined index with five parameters: number of swollen joints, number of painful joints, morning stiffness, pain visual analogue scale (VAS), and erythrocyte sedimentation rate (ESR). Functional capacity was measured by (Health Assessment Questionnaire) $H A Q$ index and the functional class was ascribed according to American College of Rheumatology criteria (1991). Articular erosion and narrowing were estimated by the modified Sharp's index. Statistical analysis was performed by chi-square, Mann-Whitney, and Kruskal-Wallis tests. A value of less 0.05 was considered significant. Results: None of the two antibodies showed association with disease flare, gender, age in the time of diagnosis, secondary Sjögren's syndrome or subcutaneous nodules. The mean age was significantly lower in $R A$ patients with positive anti-CCP. The RF and anti-CCP positivity was higher in $R A$ patients below 50 years old than patients above 50 years old. The early $R A$, up to 2 years of evolution, was not associated with a higher prevalence of anti-CCP and RF reactivity. Anti-CCP showed direct moderate correlation to $R F$ and also direct correlation to ESR and CRP. FR reactivity showed direct correlation to ESR and CRP. Functional class showed no association with neither autoantibodies. HAQ index showed correlation with anti-CCP-positive patients and activity assessed by ESR, but did not show association with disease activity, disease duration, presence of the RF and activity assessed by ESR. Sharp's

\footnotetext{
Trabalho realizado no Ambulatório de Artrite Reumatóide do Serviço de Reumatologia do Hospital Universitário Unidade Presidente Dutra da Universidade Federal do Maranhão (UFMA). Recebido em 01/12/05. Aprovado, após revisão, em 15/04/06.

1. Professor Assistente da UFMA, Mestre em Medicina Interna pela Universidade Federal de Pernambuco (UFPE), Doutorando em Medicina e Saúde pela Universidade Federal da Bahia (UFBA), Médico Assistente e Chefe do Ambulatório de Artrite Reumatóide da UFMA.

2. Professor Adjunto e Chefe da Disciplina de Reumatologia da UFMA, Mestrando em Clínica Médica pela UFMA.

3. Médica Assistente do Serviço de Reumatologia do Hospital Universitário Unidade Presidente Dutra da UFMA.

4. Professora Adjunta do Serviço de Reumatologia do Hospital Universitário Unidade Presidente Dutra da UFMA.

5. Médico Assistente e Chefe do Serviço de Radiologia do Hospital Universitário Unidade Presidente Dutra da UFMA, Chefe do Serviço de Radiologia da Santa Casa de Misericórdia de São Luís.

6. Professor Titular da Disciplina de Clínica Médica da UFBA, Chefe do Serviço de Imunologia da UFBA.

Endereço para Correspondência: Aldifran Ferreira da Silva, Rua de São Pantaleão, 300, Centro, CEP 65015-460, São Luís (MA), Brasil, tel. (98) 3222-6421 / (98) 81120348, e-mail: aldifran@ig.com.br
} 
VHS. Os índices de Sharp para erosão e pinçamento se associaram à positividade do anti-CCP. Tal associação não foi evidenciada com a positividade do FR. Conclusão: Embora o anti-CCP apresente boas propriedades diagnósticas, ele não apresenta associação com a atividade da doença, sexo, idade de início da doença, tempo de evolução de doença, presença de nódulos subcutâneos, síndrome de Sjögren ou classificação funcional do ACR de 1991. Houve associação da positividade do anti-CCP com a menor idade dos pacientes, fator reumatóide, VHS, PCR, índice de HAQ e índices de Sharp para erosão e pinçamento.

Palavras-chave: artrite reumatóide, auto-anticorpos, anti-CCP, fator reumatóide, $\mathrm{HAQ}$.

\section{INTRODUÇÃO}

A artrite reumatóide (AR) é uma doença auto-imune crônica, sistêmica, de origem desconhecida, que se caracteriza pelo envolvimento inflamatório e destrutivo de múltiplas articulações. A evolução da doença apresentando padrões distintos com diversos graus de incapacitação funcional e alterações extra-articulares, cujas diferenças individuais são marcantes, leva a importante repercussão social e econômica ${ }^{(1)}$.

Do ponto de vista prognóstico, alguns fatores, quando presentes, se associam a uma pior evolução da AR, tais como: presença de manifestações extra-articulares, fator reumatóide (FR) em altos títulos, persistência das provas de atividade inflamatória alteradas, longo tempo de doença e problemas psicossociais ${ }^{(2)}$. Todavia, a longo prazo, o prognóstico é ruim, porque $80 \%$ dos afetados estão incapacitados após 20 anos, e sua expectativa de vida é reduzida numa proporção de 3 a 18 anos $^{(3)}$. Assim, a importância de identificar variáveis que possam selecionar pacientes de pior prognóstico, candidatos a um regime terapêutico mais agressivo, vem sendo amplamente discutida na literatura.

O diagnóstico sorológico da AR tem se fundamentado, até o momento, na detecção do FR. Nas últimas décadas, limitações relativas à sua utilização, como pouca especificidade, baixa sensibilidade nos primeiros anos da doença e valor prognóstico controverso, além da necessidade atual de um diagnóstico precoce, para que possa ser instituída uma terapêutica agressiva já na fase inicial da doença ${ }^{(4-6)}$, têm motivado a pesquisa de novos testes diagnósticos e novos marcadores prognósticos para a doença.

Nos últimos anos, tem sido divulgado o valor diagnóstico e prognóstico do anticorpo antipeptídeo citrulinado cíclico (anti-CCP) na AR. Vários estudos têm demonstrado sua ocorrência precocemente no curso da doença, podendo até mesmo preceder a eclosão clínica da mesma. Ademais, erosion and narrowing index showed association to presence of anti-CCP, but not with RF positive patients. Conclusions: Although anti-CCP displays good diagnosis properties for $R A$, it appears not to be associated with activity disease, gender, age at the disease beginning, disease evolution, presence of the secondary Sjögren's syndrome and subcutaneous nodules or functional class. Anti-CCP reactivity showed association with early patients, RF positivity, ESR, CRP, HAQ index or Sharp'erosion and narrowing index.

Keywords: rheumatoid arthritis, autoantibodies, anti-CCP, rheumatoid factor, $H A Q$.

os anticorpos anti-CCP parecem estar associados à doença erosiva e progressiva ${ }^{(7-12)}$.

O presente estudo teve como objetivo analisar, numa população de pacientes com $\mathrm{AR}$, a existência de uma associação entre anti-CCP e aspectos clínicos, laboratoriais e radiográficos tradicionalmente utilizados no acompanhamento e na avaliação da gravidade da doença.

\section{PACIENTES E MÉTODOS}

\section{PACIENTES}

Participaram do presente estudo 100 pacientes consecutivos com diagnóstico de AR que foram vistos no Ambulatório de Reumatologia do Hospital Presidente Dutra da Universidade Federal do Maranhão (UFMA), no período de novembro de 2002 a junho de 2004. O critério de inclusão foi o preenchimento de, no mínimo, quatro dos sete critérios de classificação da AR, segundo o American College of Rheumatology (ACR), de $1987^{(13)}$.

O estudo foi aprovado pelo Comitê de Ética para Pesquisa em Seres Humanos do Hospital Presidente Dutra da UFMA. Após obtido o consentimento, mediante documento assinado para inclusão no estudo, todos os participantes foram submetidos a uma entrevista clínica, com ficha padronizada e coleta de aproximadamente $10 \mathrm{ml}$ de sangue de veia periférica para a realização de testes laboratoriais. As amostras de soro foram numeradas de forma aleatória para impossibilitar a identificação do paciente no momento da leitura dos resultados.

\section{PROVAS DE ATIVIDADE INFLAMATÓRIA E AUTO-ANTICORPOS}

A velocidade de hemossedimentação (VHS)- $1^{\underline{a}}$ hora - foi medida pelo método de Westergren e a proteína C reativa (PCR), pelo método imunoturbidimétrico (Roche). 
O teste do FR foi realizado pelo método do látex, segundo a técnica de imunoturbidimetria para os isótipos $\operatorname{IgM}$ (kit Spinreact). O FR foi considerado positivo quando a concentração foi maior do que o valor do ponto de corte de referência do kit $(20 \mathrm{UI} / \mathrm{ml})$.

A pesquisa do anti-CCP foi realizada pela técnica de ELISA (Immunoscan RA; EuroDiagnostica), segundo metodologia proposta por Schellekens et a ${ }^{14)}$. Amostras com resultados $\leq 25$ unidades $/ \mathrm{ml}$ foram definidas como negativas. Amostras com resultados $>25$ unidades $/ \mathrm{ml}$ foram consideradas como positivas. Pacientes com resultados $>25$ unidades $/ \mathrm{ml} \mathrm{e}<50$ unidades $/ \mathrm{ml}$ foram considerados como limítrofes.

A atividade de doença foi definida pela presença de três ou mais das seguintes condições: 1) número de articulações com calor, edema ou derrame maior ou igual a $3 ; 2$ ) número de articulações dolorosas maior ou igual a $5 ; 3$ ) tempo de rigidez matinal maior do que 30 minutos; 4) VHS na $1^{\underline{a}}$ hora maior ou igual a $30 \mathrm{~mm}$, medida pelo método de Westergren; 5) mensuração da dor, segundo a escala numérica de dor, maior do que 3. A incapacitação funcional foi medida através do Questionário de Avaliação de Saúde - HAQ (Health Assessment Questionnaire) proposto por Fries et al ${ }^{15)} \mathrm{e}$ a classe funcional dos pacientes foi determinada mediante aplicação dos critérios revisados do ACR de $1991^{(16)}$.

Radiografias de mãos e punhos dos pacientes com $\mathrm{AR}$, em incidência póstero-anterior, foram obtidas em um período de até três meses anteriores ou posteriores à avaliação clínica. Articulações de mãos e punhos foram examinadas separadamente quanto à presença de erosão e redução do espaço articular (pinçamento). O grau de lesão radiológica foi avaliado separadamente para erosão e redução do espaço articular, segundo método modificado de Sharp et a ${ }^{(17)}$. Segundo o índice de Sharp, o escore máximo previsto para erosão é igual a 170 e para pinçamento, a 144. O total de dano articular, representado pela soma desses dois valores, é igual a 314. Cada um desses valores foi representado em termos absolutos e percentuais.

Os dados foram armazenados e analisados através do software SPSS (Statistical Package for Social Sciences), versão 9.0. O teste de Kolmogorov-Smirnov foi utilizado para verificar a normalidade das variáveis contínuas. O teste do qui-quadrado ou o teste exato de Fisher foram utilizados para avaliar as diferenças das variáveis categóricas. Utilizou-se o teste t de Student e o teste de Mann-Whitney (variáveis sem distribuição normal) para comparação de duas médias das variáveis contínuas. As correlações entre as variáveis contínuas foram analisadas pelo coeficiente de correlação de Pearson ou pelo teste de Spearman (variáveis sem distribuição normal). Valores de p menores do que 5\% $(\mathrm{p}<0,05)$ foram considerados significantes.

\section{RESULTADOS}

\section{CARACTERÍSTICAS CLÍNICAS E LABORATORIAIS DOS PACIENTES COM AR}

As características clínicas e laboratoriais dos 100 pacientes reumatóides encontram-se dispostas na Tabela l. A população estudada foi constituída de 88 pacientes do sexo feminino e 12 do sexo masculino, resultando em uma razão feminino/ masculino de 7,3. A média de idade foi de $50 \pm 12,3$ anos, com amplitude de variação de 21 a 84 anos. A média de idade dos pacientes do sexo masculino foi maior que a das pacientes do sexo feminino com uma significância estatística limítrofe $(\mathrm{p}=0,06)$. O tempo de evolução da doença variou de 1 a 27 anos, com um tempo médio igual a $8 \pm 6,8$ anos.

TABELA 1

CARACTERÍSTICAS CLÍNICAS E LABORATORIAIS DOS 100 PACIENTES COM ARTRITE REUMATÓIDE

\begin{tabular}{|c|c|}
\hline Variável & $\operatorname{AR}(n=100)$ \\
\hline Sexo masculino (\%)/feminino (\%) & $12 / 88$ \\
\hline Idade (anos), média \pm DP & $50 \pm 12,3$ \\
\hline Tempo de doença (anos), média \pm DP & $8 \pm 6,8$ \\
\hline Idade de início da doença (anos), média \pm DP & $42 \pm 12,7$ \\
\hline Fase da doença: precoce (\%)/tardia (\%) & $19 / 81$ \\
\hline Doença em atividade: sim (\%)/não (\%) & $67 / 33$ \\
\hline Positividade do FR: \% & 91 \\
\hline Titulação do FR (UI/ml), média \pm DP & $124 \pm 139$ \\
\hline Positividade do anti-CCP (\%) & 68 \\
\hline Titulação do anti-CCP (UI/ml), média \pm DP & $632 \pm 786,7$ \\
\hline VHS $(\mathrm{mm} / \mathrm{h}) 1^{\mathrm{a}}$ hora, média $\pm \mathrm{DP}$ & $54 \pm 33$ \\
\hline $\mathrm{PCR}(\mathrm{mg} / \mathrm{dL})$, média $\pm \mathrm{DP}$ & $17 \pm 21$ \\
\hline Nódulos subcutâneos (\%) & 33 \\
\hline Síndrome de Sjögren (\%) & 46 \\
\hline $\begin{array}{l}\text { Classe Funcional: (\%) } \\
\text { I } \\
\text { II } \\
\text { III } \\
\text { IV }\end{array}$ & $\begin{array}{l}65 \\
25 \\
7 \\
3\end{array}$ \\
\hline $\mathrm{HAQ}$, media $\pm \mathrm{DP}($ variação) & $1,4 \pm 0,9(0-3)$ \\
\hline $\begin{array}{l}\text { Escore radiológico de Sharp, média } \pm \text { DP (variação) } \\
\text { Total } \\
\text { Erosão óssea } \\
\text { Redução do espaço articular }\end{array}$ & $\begin{array}{l}57 \pm 37(0 \text { a } 128) \\
7 \pm 10,9(0 \text { a 42) } \\
50 \pm 31,2(0-110)\end{array}$ \\
\hline
\end{tabular}

n: número de pacientes; AR: artrite reumatóide; DP: desvio padrão; FR: fator reumatóide; anti-CCP: antipeptídeo citrulinado cíclico; VHS: velocidade de hemossedimentação; PCR: proteína C reativa; HAQ: Questionário de Avaliação de Saúde. 
A idade de início da doença variou de 19 a 83 anos com idade média igual a $42 \pm 12,7$ anos, enquanto $19 \%$ $(19 / 100)$ dos pacientes encontravam-se na fase precoce da doença $(\leq 2$ anos) e $81 \%(81 / 100)$, na fase tardia (>2 anos). Segundo os parâmetros utilizados para a definição de doença em atividade, 67\% (67/100) dos pacientes encontravam-se em atividade. Em relação às outras variáveis, houve $91 \%(91 / 100)$ de positividade para o FR e títulos médios iguais a $124 \pm 139 ; 68 \%(68 / 100)$ de positividade para o anti-CCP e títulos médios iguais a $632 \pm 786,7 ; 69 \%$ $(69 / 100)$ de positividade para a VHS $(>30 \mathrm{~mm} / \mathrm{h})$ e títulos médios iguais a $54 \pm 33 ; 60 \%(60 / 100)$ de positividade para a PCR $(>5 \mathrm{mg} / \mathrm{dL})$ e títulos médios iguais a $17 \pm 21,1$; $33 \%(33 / 100)$ de presença de nódulos subcutâneos; $46 \%$ (46/100) de presença de síndrome de Sjögren. Segundo os critérios de classificação funcional da $\mathrm{AR}^{(16)}, 65 \%$ dos pacientes em estudo encontravam-se na fase I, $25 \%$ na fase II, $7 \%$ na fase III e $3 \%$ na fase IV. O valor médio do $\mathrm{HAQ}$, no conjunto de pacientes estudados, foi de $1,4 \pm$ 0,9 , com amplitude de variação de 0 a 3 . O índice médio do dano articular total pelo índice de Sharp foi de $57 \pm 37$ com amplitude de variação de 0 a 128, enquanto os valores médios com suas amplitudes de variação para erosão óssea e redução do espaço articular foram, respectivamente, $7 \pm$ $10,9(0$ a 42$)$ e $50 \pm 31,2(0$ a 110$)$.

\section{AVALIAÇÃO DO SIGNIFICADO CLÍNICO DO FR E ANTI-CCP NA ARTRITE REUMATÓIDE}

As características clínico-laboratoriais dos grupos de pacientes reumatóides de acordo com a presença ou ausência dos auto-anticorpos anti-CCP e FR encontram-se na Tabela 2. Não houve associação significante entre idade de início da AR e sexo com a presença ou ausência de qualquer um dos auto-anticorpos. A média de idade dos pacientes soropositivos para o anti-CCP e FR foi menor do que a média de idade dos pacientes soronegativos, com diferença estatisticamente significante apenas entre as médias de idade dos pacientes anti-CCP positivo e negativo. Dos 19 pacientes que se encontravam na fase precoce da doença $(\leq 2 \mathrm{anos})$, todos apresentavam positividade para o FR, enquanto $68 \%$

TABELA 2

CARACTERÍSTICAS CLÍNICO-LABORATORIAIS DOS GRUPOS DE PACIENTES REUMATÓIDES QUANTO À PRESENÇA OU AUSÊNCIA DOS AUTO-ANTICORPOS ANTI-CCP E FATOR REUMATÓIDE

\begin{tabular}{|c|c|c|c|c|c|c|}
\hline \multirow[b]{2}{*}{ VARIÁVEL } & \multicolumn{2}{|c|}{$\begin{array}{l}\text { ANTI-CCP } \\
(n=100)\end{array}$} & \multirow[b]{2}{*}{$P$} & \multicolumn{2}{|c|}{$\begin{array}{l}\text { FATOR REUMATÓIDE } \\
\qquad(\mathrm{n}=100)\end{array}$} & \multirow[b]{2}{*}{$P$} \\
\hline & $\begin{array}{l}\text { Positivo } \\
(n=68)\end{array}$ & $\begin{array}{l}\text { Negativo } \\
(n=32)\end{array}$ & & $\begin{array}{l}\text { Positivo } \\
(\mathrm{n}=91)\end{array}$ & $\begin{array}{l}\text { Negativo } \\
(\mathrm{n}=9)\end{array}$ & \\
\hline Idade de início da AR (anos), média \pm DP & $40,9 \pm 12,7$ & $44,9 \pm 12,1$ & 0,13 & $42 \pm 13,1$ & $44,1 \pm 7,7$ & 0,48 \\
\hline $\begin{array}{l}\text { Sexo } \\
\text { masculino(\%) } \\
\text { feminino }(\%)\end{array}$ & $\begin{array}{c}8(66,7) \\
60(68,2)\end{array}$ & $\begin{array}{c}4(33,3) \\
28(31,8)\end{array}$ & 1,0 & $\begin{array}{l}11(81,7) \\
80(90,9)\end{array}$ & $\begin{array}{l}1(8,3) \\
8(9,1)\end{array}$ & 1,0 \\
\hline Idade (anos), média $\pm \mathrm{DP}$ & $43,9(12,9)$ & $54(10,3)$ & $0,04^{*}$ & $50(12,5)$ & $55,7(8,4)$ & 0,12 \\
\hline $\begin{array}{l}\text { Fase da doença } \\
\text { precoce }(<2 \text { anos) }(\%) \\
\text { tardia }(\geq 2 \text { anos) }(\%)\end{array}$ & $\begin{array}{l}13(68,4) \\
55(67,9)\end{array}$ & $\begin{array}{l}6(31,6) \\
8(32,1)\end{array}$ & 0,98 & $\begin{array}{l}19(100) \\
72(88,9)\end{array}$ & $9(11,1)$ & 0,28 \\
\hline VHS $(\mathrm{mm} / \mathrm{h}) 1^{\text {a }}$ hora, média $\pm \mathrm{DP}$ & $60,2 \pm 34,1$ & $42,3 \pm 27,2$ & $0,006^{*}$ & $54,5 \pm 33,5$ & $53,8 \pm 20,8$ & 0,95 \\
\hline $\mathrm{PCR}(\mathrm{mg} / \mathrm{dL})$, média $\pm \mathrm{DP}$ & $20,9 \pm 21,4$ & $10 \pm 18,8$ & $0,01^{*}$ & $17,6 \pm 21,7$ & $14,7 \pm 10,8$ & 0,07 \\
\hline $\begin{array}{l}\text { Doença em atividade } \\
\text { sim( }(\%) \\
\text { não }(\%)\end{array}$ & $\begin{array}{l}46(68,7) \\
22(66,7)\end{array}$ & $\begin{array}{c}4(33,3) \\
28(31,8)\end{array}$ & 1,0 & $\begin{array}{l}11(81,7) \\
80(90,9)\end{array}$ & $\begin{array}{l}1(8,3) \\
8(9,1)\end{array}$ & 1,0 \\
\hline $\begin{array}{l}\text { Síndrome de Sjögren } \\
\qquad \operatorname{sim}(\%) \\
\text { não }(\%)\end{array}$ & $\begin{array}{c}34(73,9) \\
34(63)\end{array}$ & $\begin{array}{c}12(26,1) \\
20(37)\end{array}$ & 0,24 & $\begin{array}{l}41(89,1) \\
50(92,6)\end{array}$ & $\begin{array}{c}5(10,9) \\
4(7,4)\end{array}$ & 0,73 \\
\hline $\begin{array}{l}\text { Nódulos subcutâneos } \\
\qquad \operatorname{sim}(\%) \\
\text { não }(\%)\end{array}$ & $\begin{array}{c}17(89,5) \\
51(63)\end{array}$ & $\begin{array}{l}2(10,5) \\
30(37)\end{array}$ & 0,26 & $\begin{array}{l}18(94,7) \\
73(90,1)\end{array}$ & $\begin{array}{l}1(5,3) \\
8(9,9)\end{array}$ & 0,85 \\
\hline
\end{tabular}

n: número de pacientes; anti-CCP: antipeptídeo citrulinado cíclico; AR: artrite reumatóide; DP: desvio padrão; VHS: velocidade de hemossedimentação; PCR: proteína C reativa.

* Diferença significante ao nível de 5,0\%. 
(13/19) eram reagentes para o anti-CCP. A AR de início recente ( $\leq 2$ anos) não se associou a uma maior prevalência de casos soropositivos para o FR e anti-CCP, quando comparada à fase tardia da doença ( $>2$ anos de evolução).

Dos 68 pacientes que apresentavam anti-CCP reagente, 94\% (64/68) tinham FR positivo. Dos 9 pacientes com FR negativo, 44\% (4/9) apresentavam anti-CCP reagente (dados não apresentados em tabela). $\mathrm{O}$ anti-CCP apresentou uma correlação positiva moderada com o FR $(\mathrm{r}=0,44$ e $\mathrm{p}=0,01)$.

A análise das variáveis de atividade inflamatória, em função da presença ou ausência do anti-CCP, demonstrou valores médios da VHS e PCR significantemente mais elevados no grupo soropositivo (Tabela 2). Houve uma correlação direta entre o anti-CCP e a VHS $(r=0,26 \mathrm{e}$ $\mathrm{p}=0,01)$ e a PCR $(\mathrm{r}=0,32 \mathrm{e} \mathrm{p}=0,003)$. Houve, também, uma correlação direta entre o FR e a VHS $(r=0,36$ e $\mathrm{p}=0,002)$ e a PCR $(\mathrm{r}=0,26$ e $\mathrm{p}=0,02)$.

O FR foi detectado em $92 \%(62 / 67)$ e $88 \%(29 / 33)$ dos pacientes ativos e inativos, respectivamente. $\mathrm{O}$ anti-CCP foi positivo em $69 \%$ (46/67) dos ativos e 67\% (22/33) dos inativos. Não houve associação significante entre o parâmetro clínico (atividade da doença) e a presença de qualquer um dos auto-anticorpos pesquisados, assim como também em relação às manifestações extra-articulares (nódulos subcutâneos ou síndrome de Sjögren) (Tabela 2).

\section{ASSOCIAÇÃO ENTRE ANTICORPO ANTI-CCP E FR COM A GRAVIDADE DA DOENÇA}

Não foi constatada associação entre a classe funcional e a atividade ou o tempo de evolução da doença. Assim como não houve associação de qualquer um dos auto-anticorpos estudados com a classe funcional dos pacientes.

Os valores médios do HAQ foram maiores nos pacientes com positividade para os dois auto-anticorpos estudados, porém com diferença estatisticamente significante apenas para os pacientes reumatóides anti-CCP positivos (Tabela 3 ). Os níveis médios do HAQ também estiveram aumentados nos pacientes reumatóides em atividade medida pela VHS (> $30 \mathrm{~mm} / \mathrm{h})(1,52 \pm 0,89$ vs. $1,11 \pm 0,84, \mathrm{p}=0,06)$, com significância estatística limítrofe. Não houve variação estatisticamente significante do grau de incapacitação funcional em relação aos seguintes parâmetros clínico-laboratoriais da AR: atividade medida pela PCR, atividade da doença, fase da doença (precoce $\mathrm{x}$ tardia) e positividade do FR (dados não apresentados em tabela).

TABELA 3

AsSOCIAÇ̃̃o DOS AUTO-ANTICORPOS ANTI-CCP E FR EM RELAÇ̃̃o AO HAQ E O ÍNDICE RADIOLÓgico de SHARP para os 100 PaCiENTES COM AR

\begin{tabular}{|c|c|c|c|c|c|c|}
\hline \multirow[b]{2}{*}{ VARIÁVEL } & \multicolumn{2}{|c|}{$\begin{array}{l}\text { ANTI-CCP } \\
(n=100)\end{array}$} & \multirow[b]{2}{*}{$P$} & \multicolumn{2}{|c|}{$\begin{array}{l}\text { FATOR REUMATÓIDE } \\
(\mathrm{n}=100)\end{array}$} & \multirow[b]{2}{*}{$\mathrm{P}$} \\
\hline & $\begin{array}{l}\text { Positivo } \\
(\mathrm{n}=68)\end{array}$ & $\begin{array}{l}\text { Negativo } \\
(n=32)\end{array}$ & & $\begin{array}{l}\text { Positivo } \\
(\mathrm{n}=91)\end{array}$ & $\begin{array}{l}\text { Negativo } \\
(\mathrm{n}=9)\end{array}$ & \\
\hline \multicolumn{7}{|l|}{$\mathrm{HAQ}$} \\
\hline Mediana & 1,5 & 0,8 & & 1,5 & 1,4 & \\
\hline Amplitude de variação & $0-3$ & $0-2,5$ & & $0-3$ & $0,6-2,9$ & \\
\hline Média \pm DP & $1,6 \pm 0,9$ & $1,0 \pm 0,8$ & $0,007^{*}$ & $1,6 \pm 0,8$ & $1,4 \pm 0,9$ & 0,47 \\
\hline \multicolumn{7}{|c|}{ Escore radiológico de Sharp (total) } \\
\hline Mediana & 61,0 & 36,0 & & 51 & 52 & \\
\hline Amplitude de variação & $0-128$ & $0-117$ & & $0-128$ & $4-117$ & \\
\hline Média \pm DP & $63,4 \pm 35,5$ & $42,5 \pm 36,7$ & $0,017^{*}$ & $56,9 \pm 36,2$ & $53,1 \pm 48,1$ & 0,8 \\
\hline \multicolumn{7}{|l|}{$\begin{array}{l}\text { Escore radiológico de Sharp } \\
\text { para erosão }\end{array}$} \\
\hline Mediana & 4,0 & 0,8 & & 2 & 6 & \\
\hline Amplitude de variação & $0-39$ & $0-42$ & & $0-39$ & $0-42$ & \\
\hline Média $\pm \mathrm{DP}$ & $8,8 \pm 11,4$ & $2,5 \pm 8,4$ & $0,008^{*}$ & $6,3 \pm 10,2$ & $14 \pm 18$ & 0,13 \\
\hline \multicolumn{7}{|l|}{$\begin{array}{l}\text { Escore radiológico de } \\
\text { Sharp para pinçamento }\end{array}$} \\
\hline Mediana & 52,5 & 33,5 & & 47 & 52 & \\
\hline Amplitude de variação & $0-110$ & $0-104$ & & $0-110$ & $4-84$ & \\
\hline Média $\pm D P$ & $54,7 \pm 29,1$ & $40,0 \pm 33,4$ & $0,04^{*}$ & $50,6 \pm 30,9$ & $43,1 \pm 36,1$ & 0,54 \\
\hline
\end{tabular}

n: número de pacientes; anti-CCP: antipeptídeo citrulinado cíclico; DP: desvio padrão; FR: fator reumatóide; AR: artrite reumatóide.

* Diferença significante ao nível de 5,0\%. 
A análise da presença dos dois auto-anticorpos em estudo, em função das alterações radiográficas (Tabela 3), mostrou que os pacientes soropositivos para o anti-CCP, em comparação com os soronegativos, apresentaram um maior grau de erosão e de pinçamento articular, além de um maior índice total do dano articular de Sharp, com diferenças estatisticamente significantes. Não encontramos associação entre o FR e o dano articular avaliado pelo índice de Sharp. Observamos ainda, apenas em relação ao anti-CCP, uma baixa correlação positiva com o índice total de Sharp $(\mathrm{r}=0,25$ e $\mathrm{p}=0,028)$ e o grau de pinçamento $\operatorname{articular}(\mathrm{r}=0,26$ e $\mathrm{p}=0,019)$.

\section{DISCUSSÃO}

A AR é uma doença habitualmente progressiva, caracterizada por poliartrite erosiva crônica, que pode cursar com diversos graus de incapacitação funcional ${ }^{(18-19)}$. Dessa maneira, estudos que procurem identificar marcadores prognósticos de incapacidade funcional e progressão radiológica são altamente desejáveis, uma vez que são estes elementos que pretendemos evitar na história natural da doença. Neste estudo, a presença do anti-CCP em pacientes com AR está associada à maior gravidade da doença.

Muitos fatores prognósticos têm sido descritos para a $\mathrm{AR}$, como o início da doença em idade precoce e sexo feminino ${ }^{(20,21)}$. A análise de variáveis prognósticas para o anti-CCP e FR mostrou a inexistência de associação com sexo e idade de início da AR, contudo encontramos menor média de idade nos pacientes anti-CCP positivos. Fatores prognósticos como sexo e idade são de grande importância clínica, uma vez que não sofrem influência da atividade da doença e da terapêutica ${ }^{(22-24)}$. O estudo de Kroot et $a^{(8)}$ mostrou que sexo feminino e a idade do início da doença são preditores de desfecho funcional.

$\mathrm{Na}$ nossa experiência, os pacientes com $\mathrm{AR}$ na fase precoce da doença ( $\leq 2$ anos) não estão associados a uma maior prevalência de casos soropositivos para o anti-CCP quando comparados com os pacientes da fase tardia (> 2 anos). Esses resultados ocorreram, provavelmente, devido à baixa prevalência de pacientes na fase inicial da doença em nosso estudo. Na literatura, o anti-CCP tem sido apontado como um marcador diagnóstico já no início da $\mathrm{AR}^{(8,9,14)}$. Contudo, Correa et $\mathrm{a}^{(25)}$ observaram que o anti-CCP se mantém estável durante a evolução da AR.

Os testes do anti-CCP e FR-IgM são correlatos, e é considerado que a maioria dos pacientes FR-IgM positivos são também anti-CCP positivos $^{(8,14,26-30)}$. A correlação en- tre anti-CCP e FR-IgM aumenta em pacientes com $\mathrm{AR}^{(30)}$. Além disso, uma significativa percentagem de pacientes com FR-IgM (20 a 45\%) são também anti-CCP positivos ${ }^{14}$ 31-33). Em nosso estudo, $70 \%$ (64/91) dos pacientes com positividade para o FR-IgM tinham, também, anti-CCP positivo. Apesar da baixa freqüência de pacientes FR-IgM negativos em nossa casuística, observamos que 44\% (4/9) destes apresentavam reatividade para o anti-CCP. Esses dados são de grande importância, uma vez que só metade dos pacientes apresenta positividade para o FR-IgM no primeiro ano de doença. Assim, o anti-CCP foi nesse estudo, como em outros, mais hábil do que o FR em diagnosticar a doença na sua fase $\operatorname{precoce}^{(34)}$.

A sinovite pode explicar muitos dos sintomas iniciais da AR, sendo considerada como responsável pelo desenvolvimento do dano articular e invalidez. A correlação entre inflamação e dano articular tem sido bastante estudada, estando a VHS e PCR, consistentemente relacionadas com o desfecho radiológico ${ }^{(35,36-42)}$. Apesar de bem estabelecida a associação entre inflamação e dano articular, essa pode progredir mesmo diante de decréscimo da atividade inflamatória, e a erosão pode desenvolver-se em pacientes que tenham poucos sinais clínicos de inflamação. Assim, é sugerido que outros processos patológicos além da inflamação estejam envolvidos no processo destrutivo ${ }^{(43-}$ ${ }^{45)}$. Segundo os achados de Lindqvist et $\mathrm{a}^{(19)}$, o anti-CCP juntamente com a PCR foram os únicos preditores de dano articular nas mãos e pés após 10 anos de evolução; enquanto que após 5 anos os melhores preditores foram VHS e o FR-IgA. Esses dados estão em concordância com muitos outros trabalhos ${ }^{(39,42)}$. Logo, a determinação precoce desses marcadores prediz o desenvolvimento de dano articular em mãos e pés.

São escassos os estudos que têm verificado a associação entre marcadores de atividade inflamatória e anti-CCP na $\mathrm{AR}^{(46)}$. Nossos achados, assim como os de outros autores, mostram a existência de associação entre as variáveis de atividade inflamatória (VHS e PCR) e o anti-CCP ${ }^{(25,29,47)}$. A nossa amostra de pacientes anti-CCP positivos apresentam valores médios da VHS mais elevados que o grupo soronegativo, além da existência de uma correlação positiva do anti-CCP com esses marcadores inflamatórios (VHS e PCR). Assim, o anti-CCP parece ser útil no monitoramento da atividade da AR.

A maior prevalência de anti-CCP em pacientes reumatóides com doença ativa e dano articular severo sugere que o anti-CCP é bastante útil na predição do desfecho da doença ${ }^{(48,49,33)}$. De acordo com os critérios empregados 
(número de articulações inflamadas, número de articulações dolorosas, tempo de rigidez matinal, escala visual de dor e VHS), não observamos associação entre atividade da doença e anti-CCP. Esses dados confirmam os resultados de Correa et $a{ }^{(25)}$.

A presença de nódulos subcutâneos é um sinal de gravidade para a AR, sendo considerada uma importante variável preditora de dano $\operatorname{articular}^{(50)}$, estando ainda associada com o anti-CCP ${ }^{(14,51)}$. No presente estudo, assim como também relatado recentemente por Correa et $a^{(25)}$, não foi observada associação entre o anti-CCP e as manifestações extra-articulares.

A avaliação funcional dos nossos pacientes foi feita utilizando a classificação de 1991 e a dimensão do HAQ que avalia incapacidade funcional. Essas duas medidas são habitualmente empregadas para avaliação de gravidade na AR. O HAQ é considerado uma medida mais precisa que a estratificação funcional em classes $^{(52)}$. Não observamos associação entre a presença do anti-CCP ou FR e a classe funcional dos pacientes definida segundo os critérios de 1991. Nossos dados demonstram uma associação entre o HAQ e o anti-CCP, uma vez que os pacientes anti-CCP positivos apresentaram valores médios de HAQ significativamente maiores do que o grupo com anti-CCP negativo. $\mathrm{Na}$ literatura, não está bem definida a associação entre a positividade do anti-CCP e a incapacidade funcional

\section{REFERÊNCIAS}

1. Morrow J, Nelson L, Watts R, Isenberg D: Autoimmune rheumatic disease, 2 ed, 104-46 p. Oxford, Oxford University Press, 1999.

2. Chahade WH: Rheumatoid Arthritis: systemic manifestations, complications and unusual aspects. Rev Esp Reumatol 20: 144-5, 1993.

3. Albani S, Carson DA: Etiology and pathogenesis of rheumatoid arthritis. In: Koopman WJ. Arthritis and Allied Conditions (ed). A Textbook of Rheumatology. 13th ed, 979-92 p. Baltimore: Williams \& Wilkins, 1997.

4. Thorsteinsson J, Bjornsson OJ, Kolbeinsson A, Allander E, Sigfusson N, Olafsson O: A population study of rheumatoid factor in Iceland. A 5 years follow-up of 50 women with rheumatoid factor (RF). Ann Clin Res 7: 183-94, 1975.

5. Engeland T, Murthe E: The role of the laboratory in rheumatology. Rheumatoid factors. Clin Rheum Dis 9: 135-60, 1983.

6. Alessandri C, Bombardieri M, Papa N et al: Decrease of anticyclic citrullinated peptide antibodies and rheumatoid factor following anti-TNF - therapy (infliximab) in rheumatoid arthritis is associated with clinical improvement. Ann Rheum Dis 63: 1218-21, 2004.

7. Van Jaarsveld CHM, Ter Borg EJ, Jacobs JWG et al: The prognostic value of the antiperinuclear factor, anti-citrullinated peptide antibodies an rheumatoid factor in early rheumatoid arthritis. Clin Exp Rheumatol 17: 689-97, 1999. medida pelo $\mathrm{HAQ}^{(8,29,46,53,54)}$.

A análise radiográfica é considerada um dos métodos mais objetivos de avaliação da gravidade da AR. O método de quantificação do envolvimento articular descrito por Sharp et al ${ }^{17)}$, embora de mais difícil execução, é considerado o mais preciso e sensível para detecção de alterações ao longo do tempo $^{(39,55)}$. Em nosso estudo, o grau de lesão radiológica, medido através desse índice, foi significativamente maior nos pacientes anti-CCP em comparação com os soronegativos; assim como também foi maior nesse grupo de pacientes os graus de erosão e pinçamento. Essa mesma associação não foi verificada com a positividade do FR. Esses dados vêm a confirmar o valor prognóstico do anti-CCP em predizer o dano articular radiográfico $^{(7,8,19,32,33,49,53,54,56-58)}$.

A AR é retratada atualmente como uma doença progressivamente incapacitante e seu prognóstico a longo prazo tem se mostrado desfavorável numa porção significativa de $\operatorname{casos}^{(18)}$. Em face de sua heterogeneidade e potencial gravidade, faz-se necessária a identificação de variáveis cujo valor prognóstico possibilite a individualização de casos de pior evolução(19). Embora no presente trabalho tenhamos observado claramente uma correlação do anti-CCP com o HAQ (incapacidade funcional) e o índice de Sharp (destruição articular), outros estudos ainda serão necessários para confirmar o potencial valor prognóstico do anti-CCP.

8. Kroot EJJA, Jong BAW, van Leeuwen MA et al: The prognostic value of the anti-ciclic citrullinated peptide antibody in patients with recent-onset rheumatoid arthritis. Arthritis Rheum 43: 1831-5, 2000.

9. Bizzaro N, Mazzanti G, Tonutti E, Villalta D, Tozzoli R: Diagnostic accuracy of the Anti-Citrulline Antibody Assay for Rheumatoid Arthritis. Clin Chem 47: 1089-93, 2001.

10. Vencovsky J, Machacek S, Sedova L et al: Autoantibodies can be prognostic markers of an erosive disease in early rheumatoid arthritis. Ann Rheum Dis 62: 427-30, 2003.

11. Bas S, Perneger TV, Seitz M, Tiercy JM, Roux-Lombard OS, Guerne PA: Diagnostic tests for rheumatoid arthritis: comparison of anti-cyclic citrullinated peptide antibodies, anti-keratin antibodies and Ig-M rheumatoid factors. Rheumatol 41: 809-14, 2002.

12. Visser H, le Cessie S, Vos K, Breedveld FC, Hazes JM: How to diagnose rheumatoid arthritis early? A prediction model for persistent (erosive) arthritis. Arthritis Rheum 46: 357-65, 2002.

13. Arnett FC, Edworthy SM, Block DA et al: The American Rheumatism Association 1987. Revised criteria for the classification of Rheumatoid Arthritis. Arthritis Rheum 31: 315-24, 1988.

14. Schellekens GA, Visser H, de Jong BAW et al: The diagnostic properties of rheumatoid arthritis antibodies recognizing a cyclic citrullinated peptide. Arthritis Rheum 43: 155-63, 2000.

15. Fries JF, Spitz P, Kraines RG, Holman HR: Measurement of patient outcome in arthritis. Arthritis Rheum 23: 137-145, 1980. 
16. Hochberg MC, Chang RW, Dwosh I, Lindsey S, Pincus T, Wolfe F: The American College of Rheumatology 1991 revised criteria for the classification of global functional status in rheumatoid arthritis. Arthritis Rheum 35: 498-502, 1992.

17. Sharp JT, Young DY, Bluhm GB et al: How many joints in the hands and wrists should be included in a score of radiologic abnormalities used to assess rheumatoid arthritis? Arthritis Rheum 28: 1326-35, 1985

18. Markenson JA: Worldwide trends in the socioeconomic impact and long-term prognosis of rheumatoid arthritis. Semin Arthritis Rheum 21: 4-12, 1991.

19. Lindqvist E, Eberhardt K, Bendtzen K, Heinegard D, Saxne T: Prognostic laboratory markers of joint damage in rheumatoid arthritis. Ann Rheum Dis 64: 196-201, 2005.

20. Young A, van der Heijde DM: Can we predict aggressive disease? Baillierés Clin Rheumatol 11: 27-48, 1997.

21. ACR Subcommittee on Rheumatoid Arthritis Guidelines: Guidelines for the management of rheumatoid arthritis: Arthritis Rheum 46: 328-46, 2002.

22. Williamson AA, McColl GJ: Early rheumatoid arthritis: can we predict its outcome? Intern Med J 31: 168-80, 2001.

23. Symmons DP: Environmental factors and the outcome of rheumatoid arthritis, Best Pract Res Clin Rheumatol 17: 717-27, 2003.

24. Sokka T, Willoughby J, Yazici Y, Pincus T: Databases of patients with early rheumatoid arthritis in the USA. Clin Exp Rheumatol 21: S146-53, 2003.

25. Correa PA, Tobon GJ, Citera G et al: Anti-cyclic citrullinated peptide antibodies in rheumatoid arthritis: relation with clinical features, cytokines and HLA-DRB1. Biomedica 24: 140-52, 2004.

26. Goldbach-Mansky R, Lee J, McCoy A et al: Rheumatoid arthritis associated autoantibodies in patients with synovitis of recent onset. Arthritis Res 2: 236-43, 2000.

27. Us D, Gulmez D, Hascelik G: Cyclic citrullinated peptide antibodies (anti-CCP) together with some other parameters used for serologic diagnosis of rheumatoid arthritis. Mikrobiyol Bul 37: 163-70, 2003.

28. Zeng X, Ai M, Tian X et al: Diagnostic value of anti-cyclic citrullinated peptide antibody in patients with rheumatoid arthritis. J Rheumatol 30: 1451-5, 2003.

29. Kastbom A, Strandberg G, Lindroos A, Skogh T: Anti-CCP antibody test predicts the disease course during 3 years in early rheumatoid arthritis (the Swedish TIRA project). Ann Rheum Dis 63: 1085-9, 2004.

30. García-Berrocal B, González C, Pérez M et al: Anti-cyclic citrullinated peptide autoantibodies in IgM rheumatoid factorpositive patients. Clin Chim Acta 354: 123-30, 2005.

31. van Boekel MA, Vossenaar ER, van den Hoogen FH, van Venrooij WJ: Autoantibody systems in rheumatoid artritis: specificity, sensitivity and diagnostic value. Arthritis Res 4: 87$93,2002$.

32. Lee DM, Schur PH: Clinical utility of the anti-CCP assay in patients with rheumatic diseases. Ann Rheum Dis 62: 870-4, 2003.

33. Vallbracht I, Rieber J, Oppermann M, Förger F, Siebert U, Helmke K: Diagnostic and clinical value of anti-cyclic citrullinated peptide antibodies compared with rheumatoid factor isotypes in rheumatoid arthritis. Ann Rheum Dis 63: 1079-84, 2004.

34. Scott DL: Prognostic factors in early rheumatoid arthritis. Rheumatol 39: 24-9, 2000.
35. Combe B, Eliaou JF, Daures JP, Meyer O, Clot J, Sany J: Prognostic factors in rheumatoid arthritis. Comparative study of two subsets of patients according to severity of articular damage. Br J Rheumatol 34: 529-34, 1995.

36. van Leeuwen MA, van Rijswijk MH, Sluiter WJ et al: Individual relationship between progression of radiological damage and the acute phase response in early rheumatoid arthritis. Towards development of a decision support system. J Rheumatol 24: 20-7, 1997.

37. Matsuda Y, Yamanaka H, Higami K, Kashiwazaki S: Time Lag Between Active Joint Inflammation and Radiological Progression in Patients with Early Rheumatoid Arthritis. J Rheumatol 25: 427-32, 1998.

38. Graudal NA, Tarp U, Jurik AG et al: Inflammatory Patterns in Rheumatoid Arthritis Estimated by the Number of Swollen and Tender Joints, the Erythrocyte Sedimentation Rate, and Hemoglobin: Longterm Course and Association to Radiographic Progression. J Rheumatol 27: 47-57, 2000.

39. Plant MJ, Williams AL, O'Sullivan MM, Lewis PA, Coles EC, Jessop JD: Relationship between time-integrated C-reactive protein levels and radiologic progression in patients with rheumatoid arthritis. Arthritis Rheum 43: 1473-7, 2000.

40. Combe B, Dougados M, Goupille P et al: Prognostic factors for radiographic damage in early rheumatoid arthritis: a multiparameter prospective study. Arthritis Rheum 44: 173643, 2001.

41. Jansen LM, van der Horst-Bruinsma IE, van Schaardenburg D, Bezemer PD, Dijkmans BA: Predictors of radiographic joint damage in patients with early rheumatoid arthritis. Ann Rheum Dis 60: 924-7, 2001.

42. Lindqvist E, Jonsson K, Saxne T, Eberhard K: Course of radiographic damage over 10 years in a cohort with early rheumatoid arthritis. Ann Rheum Dis 62: 611-6, 2003.

43. Mulherin D, Fitzgerald O, Bresnihan B: Clinical improvement and radiological deterioration in rheumatoid arthritis: evidence that the pathogenesis of synovial inflammation and articular erosion may differ. Br J Rheumatol 35: 1263-8, 1996.

44. Kirwan JR: The relationship between synovitis and erosions in rheumatoid arthritis. Br J Rheumatol 36: 225-8, 1997.

45. van den Berg WB: Uncoupling of inflammatory and destructive mechanisms in arthritis. Semin Arthritis Rheum 30: 7-16, 2001.

46. Erre GL, Tocco A, Faedda R, Cossu A, Carcassi A: Il significato diagnostico e prognostico degli anticorpi anti-peptide citrullinato ciclico (anti-CCP) nell'artrite reumatoide. Reumatismo 56: 118-23, 2004.

47. Suzuki K, Sawada T, Murakami A et al: High diagnostic performance of ELISA detection of antibodies to citrullinated antigens in rheumatoid arthritis. Scand J Rheumatol 32: 197204,2003

48. Pinheiro GC, Scheinberg MA, Aparecida da Silva M, Maciel $S$ : Anti-cyclic citrullinated peptide antibodes in advanced rheumatoid arthritis. Ann Int Med 139: 134-5, 2003.

49. Vencovsky J, Machacek S, Sedova L et al: Autoantibodies can be prognostic markers of an erosive disease in early rheumatoid arthritis. Ann Rheum Dis 62: 427-30, 2003.

50. Meyer O, Combe B, Elias A et al: Autoantibodies predicting the outcome of rheumatoid arthritis: evaluation in two subsets of patients according to severity of radiographic damage. Ann 
Rheum Dis 56: 628-5, 1997.

51. Sebbag M, Simon M, Vincent C: The antiperinuclear factor and the so-called antikeratin antibodies are the same rheumatoid arthritisspecific autoantibodies. J Clin Invest 95: 2672-9, 1995.

52. Sherrer YS, Bloch DA, Mitchell DM, Young DY, Fries JF: The development of disability in rheumatoid arthritis. Arthritis Rheum 29: 494-500, 1986.

53. Bas S, Genevay S, Meyer O, Gabay C: Anti-cyclic citrullinated peptide antibodies, $\operatorname{IgM}$ and $\operatorname{IgA}$ rheumatoid factors in the diagnosis and prognosis of rheumatoid arthritis. Rheumatol 42: 677-80, 2003.

54. Jansen LM, van Schaardenburg D, van der Horst-Bruinsma I, van der Stadt RJ, de Koning MH, Dijkmans BA: The predictive value of anti-cyclic citrullinated peptide antibodies in early arthritis. J Rheumatol 30: 1691-5, 2003

55. Cuchacovich M, Couret M, Peray P, Gatica H, Sany J: Precision of the Larsen and Sharp methods of assessing radiologic change in patients with rheumatoid arthritis. Arthritis Rheum 35: 736-9, 1992.

56. Meyer O, Labarre C, Dougados M et al: Anticitrullinated protein/peptide antibody assays in early rheumatoid arthritis for predicting five year radiographic damage. Ann Rheum Dis. 62: $120-6,2003$

57. Bongi SM, Manetti R, Melchiorre D et al: Anti-cyclic citrullinated peptide antibodies are highly associated with severe bone lesions in rheumatoid arthritis anti-CCP and bone damage in RA. Autoimmunity 37: 495-501, 2004.

58. Forslind K, Ahlmen M, Eberhardt K, Hafstrom I, Svensson B: BARFOT Study Group. Prediction of radiological outcome in early rheumatoid arthritis in clinical practice: role of antibodies to citrullinated peptides (anti-CCP). Ann Rheum Dis 63: 1090-5, 2004. 